\title{
Modeling of the dynamics of tendon-driven robotic mechanisms with flexible tendons
}

\author{
Yueh-Hua Lee, Jyh-Jone Lee * \\ Department of Mechanical Engineering, National Taiwan University, Taipei 106, Taiwan, ROC
}

Received 12 September 2002; accepted 20 March 2003

\begin{abstract}
In this paper, a systematic methodology for the dynamic analysis of tendon-driven robotic mechanisms with compliant tendons is presented. The compliance of tendons and inertias of the intermediate links in the mechanism are taken into account. Representing the tendon force by use of a rectifying operator, the unidirectional force transmission characteristic of tendons can be preserved. The dynamic equations can then be systematically established in a recursive manner using the Newton-Euler equations. The joint reaction forces and the tension in each segment of tendon can be also obtained. The methodology can be applied to both endless and open-ended tendon-driven robotic mechanisms.
\end{abstract}

(c) 2003 Elsevier Ltd. All rights reserved.

Keywords: Dynamic analysis; Tendon-driven; Compliance; Recursive; Newton-Euler equations

\section{Introduction}

For decades, the robotic manipulators using tendon transmission have attracted researchers for applications in many areas such as the design of dexterous mechanical hands [1,2], parallel cablesuspended manipulators [3,4], teleoperating robots, etc. A unique characteristic associated with the robotic manipulator using tendon transmission lies in that tendons can only exert tension, i.e., force is only transmitted in a unidirectional sense. As a result, it increases the complexity in the control of such mechanical systems. The problem can be further complicated by friction and compliance embedded in the tendons and/or inertia of the extra components used in the systems.

\footnotetext{
* Corresponding author. Tel.: +886-2-2364-8501; fax: +886-2-2363-1755.

E-mail address: jjlee@ccms.ntu.edu.tw (J.-J. Lee).
} 


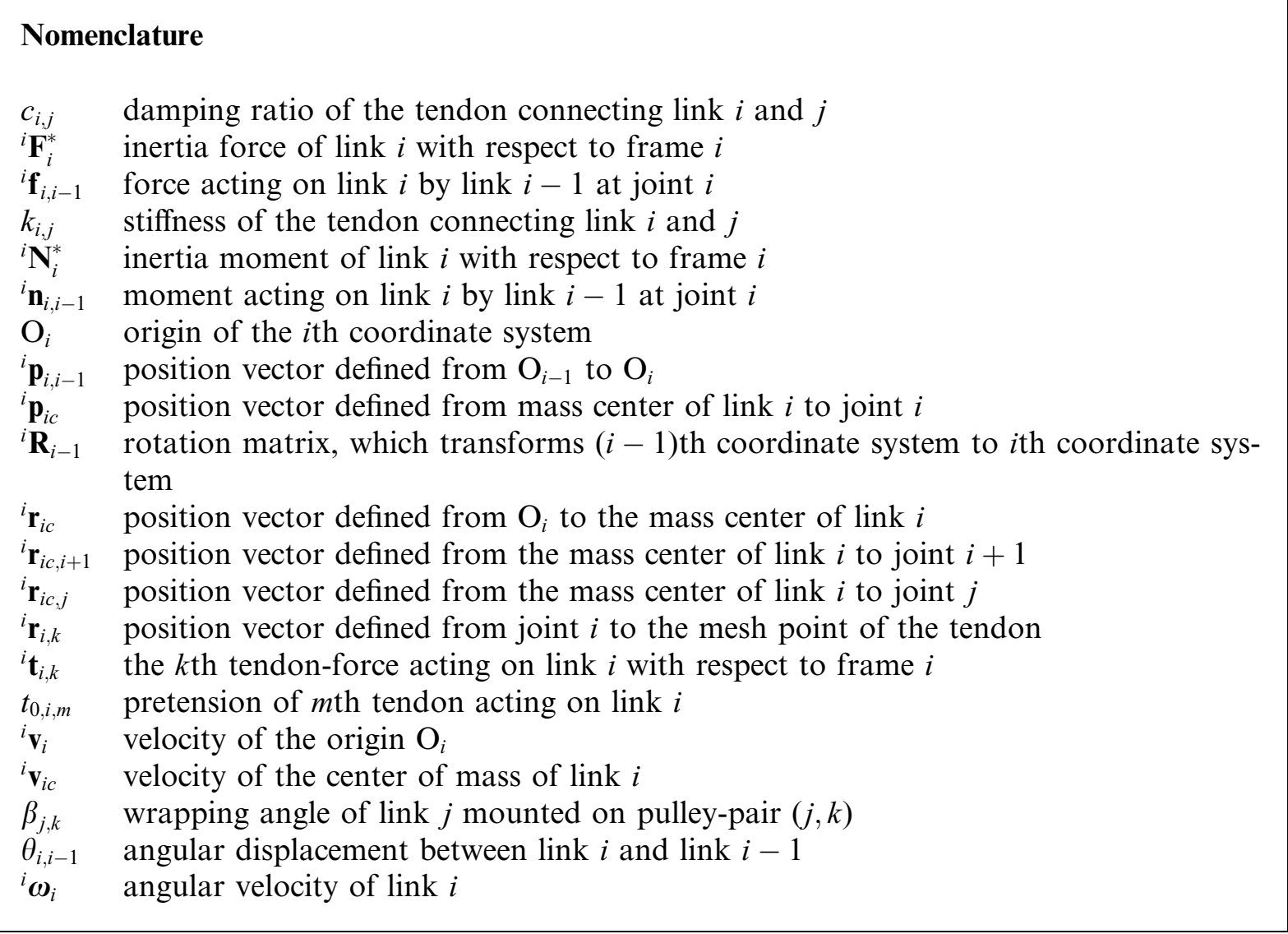

Therefore, it would be useful to research on the development of an analytical model that can account for a number of their realistic properties, such as tendon compliance, link inertia, and/or joint friction in order to produce predictions that are important for design. Also, it can provide the means for the engineer to realize the performance of such mechanical systems for future extent.

A number of analytic modeling methods for tendon-driven systems have been proposed and studied. The kinematics and statics of articulated tendon-driven robotic mechanisms have been investigated by Morecki et al. [5], Salisbury [1] and Tsai and Lee [6]. Ideal tendons with no compliance were used in their studies. Hollars and Cannon [7] experimented on the control of a two-link manipulator with flexible tendons. They concluded that the flexibility in drive trains has a significant influence on the fine position control of the tendon-driven system. Chang [8] used the stiffness constant to represent the stiffness of tendons and studied the compliance of tendon-driven robotic mechanisms. Prisco and Bergamasco [9] derived the dynamics of a type $(2 N)$ of multidegree-of-freedom (multi-DOF) tendon-driven manipulators, which the structure Utah-MIT hand [2] belongs to. On the other hand, there is also some literature investigating on the performance of single-DOF tendon devices $[10,11]$ of which the kinematic structure is less coupled than that of the multi-DOF system. 
In this paper, a systematic methodology for generating the dynamic model of robotic mechanisms with compliant tendons is presented. A model is first developed for representing the unidirectional force transmission characteristic of tendons. The resulting representation is then combined with the Newton-Euler algorithm to generate the system dynamic equations. The procedure is shown by the application of the method to a two-link tendon-driven robotic manipulator and a one-DOF manipulator equipped with a simple servo-control. The results show that the unique characteristic of tendon transmission can be preserved in the modeling. They also show that the modeling will be degraded if the compliance associated with position control is not properly managed.

\section{Assumptions}

In [6], some general assumptions about the structural characteristics have been introduced for the kinematic and static analysis of tendon-driven manipulators. To adapt to the scope of dynamic analysis, the following assumptions for tendon-driven manipulators are considered:

(I) All tendons are under tension. The amount of stretch in tendon is small and linearly proportional to the tendon force.

(II) No slippage occurs between pulleys and tendons.

(III) Tendons are lightweight such that the weight/inertia, flexural bending, and shear effects of tendons will not be considered.

(IV) For sakes of clarity and simplicity, the frictions in the pulley bearings and other moving parts of the transmission are not included. Nevertheless, these terms may be added in the modeling where the force control is important.

Tendon drives can generally be classified into two types of routing [6,12] as (1) the open-ended type and (2) the endless type of routing. In the open-ended tendon routing, as shown in Fig. 1a, one end of the tendon is fixed to a moving link to be controlled while the other end is attached to a driving actuator. From the moving link to the driving actuator, each tendon routing forms a transmission line. A unique characteristic of such open-ended tendon drives is that tendons transmit the forces in a unidirectional sense. On the other hand, the routing of the endless tendons is shown in Fig. $1 \mathrm{~b}$ in which two pulleys of constant center distance are wrapped around by an endless tendon. The driven pulley is attached to a link to be controlled and the driving pulley is installed on a rotary actuator or fixed to a driven pulley of prior pulley train stage. From the driven link to the driving actuator, the tendon-and-pulley also forms a transmission line. In an endless tendon drive, the pulley can be driven in both directions. One side of the tendon will be under higher tension while the other side is under lower tension.

It can be noted that after the removal of tendons and intermediate/idle pulleys, the tendondriven robotic mechanism becomes a serial type open-loop chain. We call the links that constitute the open-loop chain as the primary links, and all other links as the intermediate links. An intermediate link is said to be carried by a primary link $i$ if it is connected to link $i$ by a revolute joint. As shown in Fig. 1a, links 0, 1, 2 and 3 are the primary links, and links 4, 5 are the intermediate links. Intermediate links 4 and 5 are carried by the primary link 1 . 

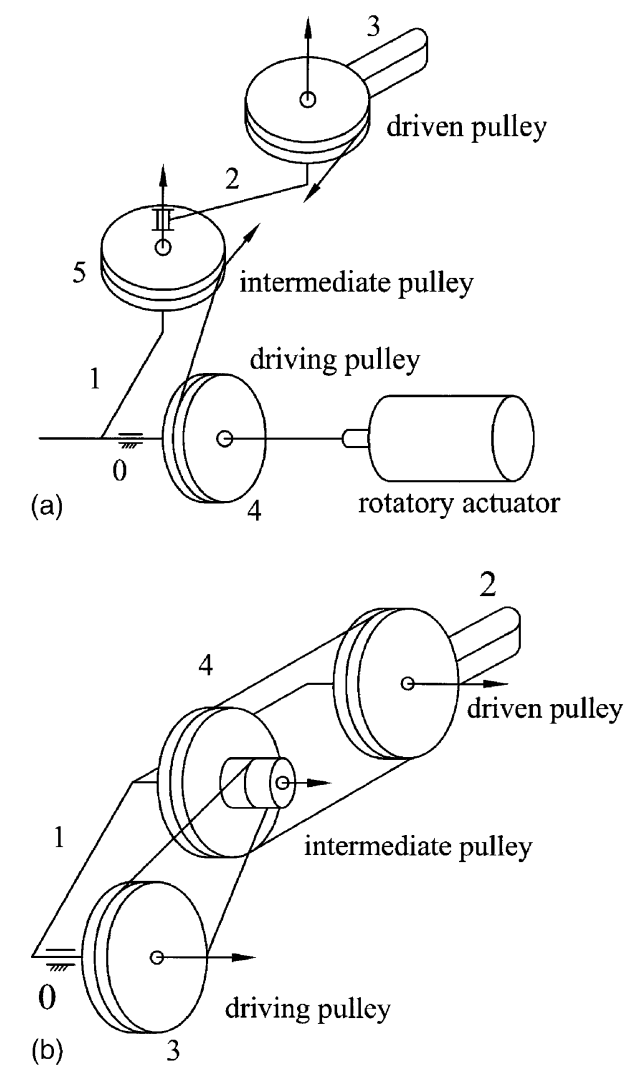

Fig. 1. (a) Open-ended type and (b) endless type tendon routing.

\section{Kinematics}

\subsection{Kinematics of primary links}

To facilitate the analysis, each primary link from the base to the distal link is sequentially numbered from 0 to $n$. Meanwhile, a local coordinate system $\left(x_{i}, y_{i}, z_{i}\right)$ is attached to the distal joint of link $i$ according to the Denavit and Hartenberg (D-H) convention [13]. Let $\theta_{i, i-1}$ be the joint angle from $x_{i-1}$ axis to $x_{i}$ axis, $a_{i, i-1}$ and $\alpha_{i, i-1}$ be the offset distance and the twist angle between $z_{i-1}$ and $z_{i}$ axes, and $d_{i, i-1}$ be the translational distance measured from $x_{i-1}$ axis to $x_{i}$ axis along $z_{i-1}$ axis. Then, the matrix transforming the $i$ th coordinate to the $(i-1)$ th coordinate can be written as

$$
{ }^{i-1} \mathbf{A}_{i}=\left[\begin{array}{cccc}
C \theta_{i, i-1} & -C \alpha_{i, i-1} S \theta_{i, i-1} & S \alpha_{i, i-1} S \theta_{i, i-1} & a_{i, i-1} C \theta_{i, i-1} \\
S \theta_{i, i-1} & C \alpha_{i, i-1} C \theta_{i, i-1} & -S \alpha_{i, i-1} C \theta_{i, i-1} & a_{i, i-1} S \theta_{i, i-1} \\
0 & S \alpha_{i, i-1} & C \alpha_{i, i-1} & d_{i, i-1} \\
0 & 0 & 0 & 1
\end{array}\right]
$$

where $C \theta_{i, i-1}=\cos \left(\theta_{i, i-1}\right), S \theta_{i, i-1}=\sin \left(\theta_{i, i-1}\right), C \alpha_{i, i-1}=\cos \left(\alpha_{i, i-1}\right)$, and $S \alpha_{i, i-1}=\sin \left(\alpha_{i, i-1}\right)$. 
The velocities and accelerations of the primary links can be derived from the forward recursive method [14], computing from the proximal moving link toward the end-effector link, as

$$
\begin{aligned}
{ }^{i} \boldsymbol{\omega}_{i} & ={ }^{i} \mathbf{R}_{i-1}\left({ }^{i-1} \boldsymbol{\omega}_{i-1}+\dot{\theta}_{i, i-1}{ }^{i-1} \mathbf{z}_{i-1}\right) \\
{ }^{i} \dot{\boldsymbol{\omega}}_{i} & ={ }^{i} \mathbf{R}_{i-1}\left({ }^{i-1} \dot{\boldsymbol{\omega}}_{i-1}+\ddot{\theta}_{i, i-1}{ }^{i-1} \mathbf{z}_{i-1}+{ }^{i-1} \boldsymbol{\omega}_{i-1} \times \dot{\theta}_{i, i-1}{ }^{i-1} \mathbf{z}_{i-1}\right) \\
{ }^{i} \mathbf{v}_{i} & ={ }^{i} \mathbf{R}_{i-1}{ }^{i-1} \mathbf{v}_{i-1}+{ }^{i} \boldsymbol{\omega}_{i} \times{ }^{i} \mathbf{p}_{i, i-1} \\
{ }^{i} \dot{\mathbf{v}}_{i} & ={ }^{i} \dot{\boldsymbol{\omega}}_{i} \times{ }^{i} \mathbf{p}_{i, i-1}+{ }^{i} \mathbf{R}_{i-1}{ }^{i-1} \dot{\mathbf{v}}_{i-1}+{ }^{i} \boldsymbol{\omega}_{i} \times\left({ }^{i} \boldsymbol{\omega}_{i} \times{ }^{i} \mathbf{p}_{i, i-1}\right) \\
{ }^{i} \dot{\mathbf{v}}_{i c} & ={ }^{i} \dot{\boldsymbol{\omega}}_{i} \times{ }^{i} \mathbf{r}_{i c}+{ }^{i} \dot{\mathbf{v}}_{i}+{ }^{i} \boldsymbol{\omega}_{i} \times\left({ }^{i} \boldsymbol{\omega}_{i} \times{ }^{i} \mathbf{r}_{i c}\right)
\end{aligned}
$$

where $\omega_{i}$ is the angular velocity vector of link $i, \mathbf{v}_{i}$ is the velocity vector of the origin $\mathrm{O}_{i}, \mathbf{v}_{i c}$ is the velocity of the center of mass of link $i, \mathbf{r}_{i c}$ is the position vector defined from the mass center of link $i$ to $\mathrm{O}_{i},{ }^{i-1} \mathbf{z}_{i-1}=[0,0,1]^{\mathrm{T}}$ is a unit vector defined along the $\mathbf{z}_{i-1}$ axis, ${ }^{i} \mathbf{R}_{i-1}$ is the transpose of the upper left $3 \times 3$ sub-matrix of ${ }^{i-1} \mathbf{A}_{i}$, and ${ }^{i} \mathbf{p}_{i, i-1}$ is the vector as

$$
{ }^{i} \mathbf{p}_{i, i-1}=\left[a_{i, i-1}, d_{i, i-1} S \alpha_{i, i-1}, d_{i, i-1} C \alpha_{i, i-1}\right]^{\mathrm{T}}
$$

\subsection{Kinematics of intermediate links}

A local coordinate system $\left(x_{j}, y_{j}, z_{j}\right)$ is defined for each intermediate link according to the D-H convention. Consider an intermediate link $j$ located on the primary link $i$, the coordinate transformation matrix from the $j$ th coordinate system to the $i$ th coordinate is given by

$$
{ }^{i} \mathbf{B}_{j}=\left[\begin{array}{cccc}
C \theta_{j, i} & -C \alpha_{j, i} S \theta_{j, i} & S \alpha_{j, i} S \theta_{j, i} & a_{j, i} C \theta_{j, i} \\
S \theta_{j, i} & C \alpha_{j, i} C \theta_{j, i} & -S \alpha_{j, i} C \theta_{j, i} & a_{j, i} S \theta_{j, i} \\
0 & S \alpha_{j, i} & C \alpha_{j, i} & d_{j, i} \\
0 & 0 & 0 & 1
\end{array}\right]
$$

Suppose links $i, j$, and $k$ are coaxial. Then, the relative motion among these links can be related by the coaxial condition [6] as

$$
\theta_{i, k}=\theta_{i, j}+\theta_{j, k}
$$

The velocity and acceleration of intermediate link $j$ can be also obtained by modifying Eqs. (2)(6) as

$$
\begin{aligned}
& { }^{j} \boldsymbol{\omega}_{j}={ }^{j} \mathbf{Q}_{i}^{i} \boldsymbol{\omega}_{i}+\dot{\theta}_{j, i}{ }^{j} \mathbf{z}_{j} \\
& { }^{j} \dot{\boldsymbol{\omega}}_{j}={ }^{j} \mathbf{Q}_{i}^{i} \dot{\boldsymbol{\omega}}_{i}+\ddot{\theta}_{j, i}{ }^{j} \mathbf{z}_{j}+{ }^{j} \mathbf{Q}_{i}^{i} \boldsymbol{\omega}_{i} \times \dot{\theta}_{j, i}{ }^{j} \mathbf{z}_{j} \\
& { }^{j} \mathbf{v}_{j}={ }^{j} \mathbf{Q}_{i}^{i} \mathbf{v}_{i}+{ }^{j} \boldsymbol{\omega}_{j} \times{ }^{j} \mathbf{p}_{j, i} \\
& { }^{j} \dot{\mathbf{v}}_{j}={ }^{j} \dot{\boldsymbol{\omega}}_{j} \times{ }^{j} \mathbf{p}_{j, i}+{ }^{j} \mathbf{Q}_{i}^{i} \dot{\mathbf{v}}_{i}+{ }^{j} \mathbf{Q}_{i}^{i} \boldsymbol{\omega}_{i} \times\left({ }^{j}{ }^{j} \boldsymbol{\omega}_{j} \times{ }^{j} \mathbf{p}_{j, i}\right) \\
& { }^{j} \dot{\mathbf{v}}_{j c}={ }^{j} \mathbf{Q}_{i}^{i} \dot{\boldsymbol{\omega}}_{i} \times\left({ }^{j} \mathbf{p}_{j, i}+{ }^{j} \mathbf{r}_{j c}\right)+{ }^{j} \mathbf{Q}_{i}^{i} \dot{\mathbf{v}}_{i}+{ }^{j} \mathbf{Q}_{i}^{i} \boldsymbol{\omega}_{i} \times\left[{ }^{j} \mathbf{Q}_{i}^{i} \boldsymbol{\omega}_{i} \times\left({ }^{j} \mathbf{p}_{j, i}+{ }^{j} \mathbf{r}_{j c}\right)\right]
\end{aligned}
$$

where ${ }^{j} \mathbf{Q}_{i}$ is the transpose of the upper left $3 \times 3$ submatrix of ${ }^{i} \mathbf{B}_{j}, \mathbf{r}_{j c}$ is the position vector defined from mass center of links $j$ to $\mathrm{O}_{j}$, and ${ }^{j} \mathbf{p}_{j, i}$ can be written as 


$$
{ }^{j} \mathbf{p}_{j, i}=\left[a_{j, i}, d_{j, i} S \alpha_{j, i}, d_{j, i} C \alpha_{j, i}\right]^{\mathrm{T}}
$$

\section{Modeling of the unidirectional force in tendon}

A number of models for the dynamics of tendon-driven robotic manipulators with elastic tendons have been developed $[7,8,15]$. In the models, the elastic tendon connecting two pulleys is represented by a linear spring. Since linear springs can exert tensile as well as compressive force, therefore, modeling the tendon with linear spring will inevitably be biased from the realistic characteristics of tendon. In this work, a method for modeling the unidirectional force in the tendon is proposed. The method utilizes the rectifying concept that was used by Jacobsen et al. [2] and Lee and Tsai [16]. Jacobsen et al. ever used a rectifying operator to transform the joint torque space into tendon force (or motor torque) space for the Utah-MIT dexterous hand. Lee and Tsai further extended the concept to motor torque analysis for the $n \times(n+1)$ tendon-driven systems. In what follows, the basic principle of such concept will be first introduced. Then, the procedure of the application for the tendon force analysis will be developed. Let the operator $\mathrm{O}^{+}(x)$ be defined as

$$
\mathrm{O}^{+}(x)= \begin{cases}x, & x \geqslant 0 \\ 0, & x<0\end{cases}
$$

where $x$ is a dummy variable. The function of $\mathrm{O}^{+}(x)$ can be plotted graphically as shown in Fig. 2. Also, $\mathrm{O}^{+}(x)$ can be written as

$$
\mathrm{O}^{+}(x)=(x+|x|) / 2
$$

It can be noted that $\mathrm{O}^{+}(x)$ returns its value only when the argument is positive. Otherwise, $\mathrm{O}^{+}(x)$ gives zero value.

Fig. 3 shows the planar schematic of a simple tendon-and-pulley pair, where link $k$ is the carrier and links $i$ and $j$ are the two pulleys. Pulley $i$ and $j$ are coupled by a flexible tendon having the spring constant $k_{i, j}$ and damping coefficient $c_{i, j}$. If the positive rotation of pulley is defined as the pulley rotates counterclockwise, then, for small angular rotations of the pulleys, the magnitude of tendon force, $t_{i, j}$, can be written as

$$
t_{i, j}=\mathrm{O}^{+}\left[t_{0, i, j}-k_{i, j}\left(r_{i} \theta_{i, k}-r_{j} \theta_{j, k}\right)-c_{i, j}\left(r_{i} \dot{\theta}_{i, k}-r_{j} \dot{\theta}_{j, k}\right)\right]
$$

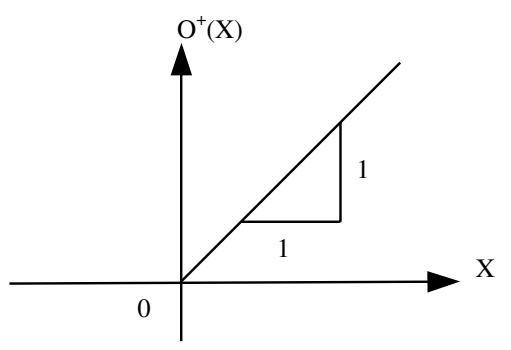

Fig. 2. Function of $\mathrm{O}^{+}(x)$. 


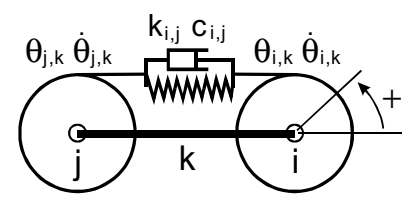

Fig. 3. A parallel-type routing pulley pair.

where $t_{0, i, j}$ is the amount of pretension that is initially set in the tendon. The physical meaning of Eq. (18a) can be readily seen from Fig. 3. If the value in the square bracket is greater than zero, then the tendon is under tension and the operator returns the value. On the other hand, if the value in the square bracket is less than zero, $t_{i, j}$ is equal to zero and the tendon becomes slack. Therefore, the equation implies that the tendon force can be transmitted only when the relative displacement between two pulleys causes tendon under tension. In general, to satisfy different types of pair connections, Eq. (18a) can be rewritten as

$$
t_{i, j}=\mathrm{O}^{+}\left[t_{0, i, j} \pm k_{i, j} r_{i} \theta_{i, k} \pm k_{i, j} r_{j} \theta_{j, k} \pm c_{i, j} r_{i} \dot{\theta}_{i, k} \pm c_{i, j} r_{j} \dot{\theta}_{j, k}\right]
$$

where the \pm sign of each term is to be determined by the tendon routing topology. The sign is positive if the pulley tends to pull tendon from the other end; otherwise, it is negative.

\subsection{Tendon forces analysis}

Since each transmission line begins from the primary link and ends at the link driven by the rotary actuator, the tendon may route through one or more intermediate links. Consider the intermediate link $j$ on the distal joint of a primary link $i-1$ as shown in Fig. 4. Let the carrier of the pulley pair $(j, j+1)$ be the primary link $i$, and the carrier of the pulley pair $(j, j-1)$ be the primary link $i-1$. Also, let the portion of the tendon connecting the intermediate links $j$ and $j+1$ be $\mathbf{t}_{j, j+1}$, and the other portion connecting links $j$ and $j-1$ be $\mathbf{t}_{j, j-1}$.

For a general tendon-pulley pair, the tendon will engage with the pulley at a constant direction with respect to the carrier. In addition, for the pulley to run in both directions, the direction of

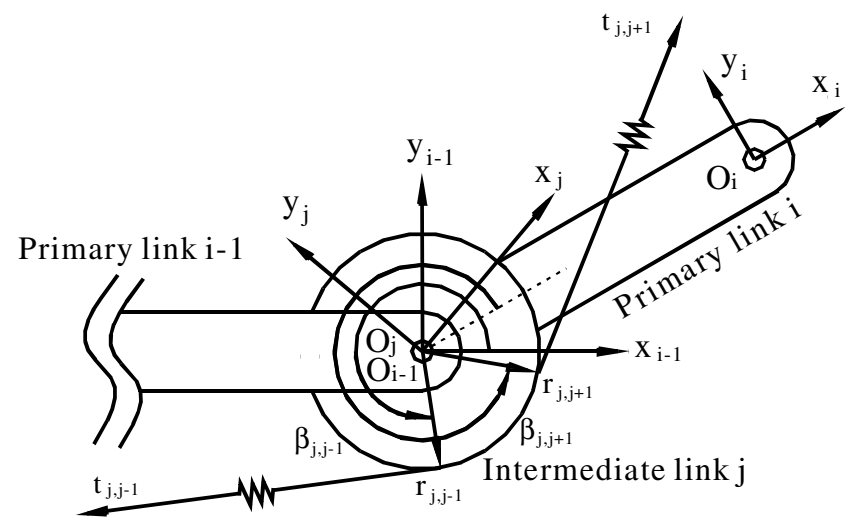

Fig. 4. A typical intermediate link $j$ carried by a primary link $i-1$. 
tendon acting on the pulley should be tangential to the pitch circle of the pulley plane. As shown in Fig. 4, define the radius vector $\mathbf{r}_{j, j+1}$ of tendon $\mathbf{t}_{j, j+1}$ as the vector from the axis of rotation to the tangent point, and the wrapping angle $\beta_{j, j+1}$ as the angle measured from the $x$-axis of coordinate system on link $i$ to the radius vector in a right-hand rule direction. Note that in the spatial case, the $x$-axis is defined by the coordinate systems of the pulley $j$ and link $i$ according to the $\mathrm{D}-\mathrm{H}$ method. Similarly, the radius vector, $\mathbf{r}_{j, j-1}$, and the wrapping angle, $\beta_{j, j-1}$, for tendon $\mathbf{t}_{j, j-1}$ can be defined in the $(i-1)$ th system. The radii vectors and tendon forces are summarized as

$$
\begin{aligned}
& { }^{i} \mathbf{r}_{j, j+1}={ }^{i} \mathbf{G}_{i-1} \cdot\left[\begin{array}{lll}
r_{j} C \beta_{j, j+1} & r_{j} S \beta_{j, j+1} & b_{j, i+1}
\end{array}\right]^{\mathrm{T}} \\
& { }^{i-1} \mathbf{r}_{j, j-1}=\left[\begin{array}{lll}
r_{j} C \beta_{j, j-1} & r_{j} S \beta_{j, j-1} & b_{j, i-1}
\end{array}\right]^{\mathrm{T}} \\
& { }^{i} \mathbf{t}_{j, j+1}=t_{j, j+1}{ }^{i} \mathbf{u}_{j, j+1} \\
& { }^{i-1} \mathbf{t}_{j, j-1}=t_{j, j-1}{ }^{i-1} \mathbf{u}_{j, j-1}
\end{aligned}
$$

where $r_{j}$ is the radius of link $j, b_{j, i+1}$ is the distance measured from origin $\mathrm{O}_{i}$ to the center of pulley $j$ along $z_{j}, b_{j, i-1}$ is the distance measured from origin $\mathrm{O}_{i-1}$ to the center of pulley $j$ along $z_{j-1}, t_{j, j+1}$ and $t_{j, j-1}$ are respectively the magnitudes of $\mathbf{t}_{j, j+1}$ and $\mathbf{t}_{j, j-1}$, which can be formulated form Eq. (18b). Vectors ${ }^{i} \mathbf{u}_{j, j+1}$ and ${ }^{i-1} \mathbf{u}_{j, j-1}$ are respectively the unit vectors of ${ }^{i} \mathbf{t}_{j, j+1}$ and ${ }^{i-1} \mathbf{t}_{j, j-1}$ and can be written as

$$
\begin{aligned}
& { }^{i} \mathbf{u}_{j, j+1}={ }^{i} \mathbf{G}_{i-1} \cdot\left[\begin{array}{lll}
-S \beta_{j, j+1} & +C \beta_{j, j+1} & 0
\end{array}\right]^{\mathrm{T}} \\
& { }^{i-1} \mathbf{u}_{j, j-1}=\left[\begin{array}{lll}
+S \beta_{j, j-1} & -C \beta_{j, j-1} & 0
\end{array}\right]^{\mathrm{T}}
\end{aligned}
$$

The transformation matrix ${ }^{i} \mathbf{G}_{i-1}$ is defined as:

$$
{ }^{i} \mathbf{G}_{i-1}=\left[\begin{array}{ccc}
1 & 0 & 0 \\
0 & C \alpha_{i, i-1} & S \alpha_{i, i-1} \\
0 & -S \alpha_{i, i-1} & C \alpha_{i, i-1}
\end{array}\right]
$$

where $\alpha_{i, i-1}$, defined in $\mathrm{D}-\mathrm{H}$ notation, is the twist angles between the $z_{j}$ and $z_{i}$ axes.

The tendon forces can be transformed to the $j$ th coordinate system on link $j$ by multiplying a suitable rotational matrix ${ }^{j} \mathbf{R}_{i-1}$.

$$
\begin{aligned}
{ }^{j} \mathbf{t}_{j, j+1} & ={ }^{j} \mathbf{R}_{i}{ }^{i} \mathbf{t}_{j, j+1} \\
{ }^{j} \mathbf{t}_{j, j-1} & ={ }^{j} \mathbf{R}_{i-1}{ }^{i-1} \mathbf{t}_{j, j-1}
\end{aligned}
$$

\section{Dynamic analysis}

For a given mechanism, the force and moments exerted by different links on each other can be determined via Newton and Euler's equations. While solving the dynamics using Newton and Euler's equations, three force- and three moment-balance equations can be established for each link of the mechanism. To solve the simultaneous equations in an efficient way, rather than solving all the unknowns simultaneously, one tries to solve them in a recursive manner. The joint forces will first be expressed in the state of motions, and then the state of motions will be obtained 
by solving the differential equations. After the state of motions is solved, the joint forces can then be determined by back substituting the tendon forces into the balance equations.

\subsection{Dynamics of primary links}

Fig. 5 shows the free body diagram of a primary link $i$ which connects to link $(i-1)$ at joint $(i-1)$ and link $(i+1)$ at joint $i$. Assume that primary link $i$ is driven by an $m$ th tendon (or more). The force and moment balance equations for link $i$ can be represented in the following recursive forms:

$$
\begin{aligned}
{ }^{i} \mathbf{F}_{i}^{*}= & { }_{i, i-1}^{i}+{ }^{i} \mathbf{f}_{i, i+1}+\sum_{m} \mathrm{O}^{+}\left(t_{0, i, m} \pm k_{i, m} r_{i} \theta_{i, i-1} \pm k_{i, m} r_{m} \theta_{m, i-1} \pm c_{i, m} r_{i} \dot{\theta}_{i, i-1} \pm c_{i, m} r_{m} \dot{\theta}_{m, i-1}\right)^{i} \mathbf{u}_{i, m} \\
& -\sum_{j}{ }^{i} \mathbf{f}_{j, i}
\end{aligned}
$$

and

$$
\begin{aligned}
{ }^{i} \mathbf{N}_{i}^{*}= & { }^{i} \mathbf{n}_{i, i-1}+{ }^{i} \mathbf{n}_{i, i+1}+{ }^{i} \mathbf{p}_{i c} \times{ }^{i} \mathbf{f}_{i, i-1}+{ }^{i} \mathbf{r}_{i c, i+1} \times{ }^{i} \mathbf{f}_{i, i+1}+\sum_{j}{ }^{i} \mathbf{n}_{i, j}-\sum_{j}\left({ }^{i} \mathbf{r}_{i c, j} \times{ }^{i} \mathbf{f}_{j, i}\right) \\
& +\sum_{m}\left[\left({ }^{i} \mathbf{r}_{i, m}+{ }^{i} \mathbf{p}_{i c}\right) \times \mathrm{O}^{+}\left(t_{0, i, m} \pm k_{i, m} r_{i} \theta_{i, i-1} \pm k_{i, m} r_{m} \theta_{m, i-1} \pm c_{i, m} r_{i} \dot{\theta}_{i, i-1} \pm c_{i, m} r_{m} \dot{\theta}_{m, i-1}\right){ }^{i} \mathbf{u}_{i, m}\right]
\end{aligned}
$$

where ${ }^{i} \mathbf{u}_{i, m}$ is the unit vector of $\mathbf{t}_{i, m}$ acting on link $i$. The vector ${ }^{i} \mathbf{u}_{i, m}$ can be determined from Eqs. (20c) and (20d). ${ }^{i} \mathbf{F}_{i}^{*}$ and ${ }^{i} \mathbf{N}_{i}^{*}$ are the inertia force and moment vectors of primary link $i$, and can be obtained as:

$$
\begin{aligned}
{ }^{i} \mathbf{F}_{i}^{*} & =m_{i}^{i} \dot{\mathbf{v}}_{i c} \\
{ }^{i} \mathbf{N}_{i}^{*} & ={ }^{c} \mathbf{I}_{i}{ }_{i} \dot{\boldsymbol{\omega}}_{i}+{ }^{i} \boldsymbol{\omega}_{i} \times\left({ }^{c} \mathbf{I}_{i}{ }_{i} \boldsymbol{\omega}_{i}\right)
\end{aligned}
$$

where $m_{i}$ is the mass of link $i$, and ${ }^{c} \mathbf{I}_{i}$ is the inertia tensor of link $i$ with respect to a center of mass coordinate system, which has the same orientation as the $i$ th coordinate system.

The vector terms in Eqs. (23a) and (23b), $\mathbf{f}_{i+1, i}$ and $\mathbf{n}_{i+1, i}$, are computed from the balance equations of the preceding link while $\mathbf{f}_{j, i}$ and $\mathbf{n}_{j, i}$ are the forces and moments from intermediate links. For the end-effector link, these vectors represent the end-effector output force and moment. The other unknown terms: $\mathbf{f}_{i, i-1}, \mathbf{n}_{i, i-1}, \theta_{i, i-1}$, and $\theta_{m, i-1}$ always constitute more than six scalar unknowns. Hence they cannot be solved one link at a time.

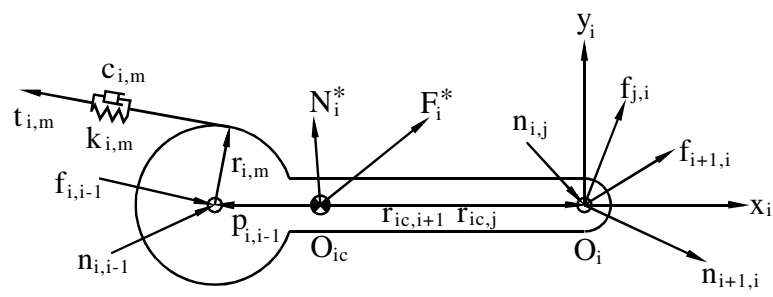

Fig. 5. A typical primary link $i$. 


\subsection{Dynamics of intermediate links}

Referring to Fig. 6 the intermediate link $j$ is located on link $i$, the force and moment balance equations for an intermediate link $j$ can be written as

$$
\begin{gathered}
{ }^{j} \mathbf{F}_{j}^{*}={ }_{j}^{j} \mathbf{f}_{j, i}+\mathrm{O}^{+}\left(t_{0, j, m} \pm k_{j, m} r_{j} \theta_{j, i} \pm k_{j, m} r_{m} \theta_{m, i} \pm c_{j, m} r_{j} \dot{\theta}_{j, i} \pm c_{j, m} r_{m} \dot{\theta}_{m, i}\right)^{j} \mathbf{u}_{j, m} \\
+\mathrm{O}^{+}\left(t_{0, j, m+1} \pm k_{j, m+1} r_{j} \theta_{j, i+1} \pm k_{j, m+1} r_{m+1} \theta_{m+1, i+1} \pm c_{j, m+1} r_{j} \dot{\theta}_{j, i+1}\right. \\
\left.\quad \pm c_{j, m+1} r_{m+1} \dot{\theta}_{m+1, i+1}\right)^{j} \mathbf{u}_{j, m+1}
\end{gathered}
$$

and

$$
\begin{aligned}
{ }^{j} \mathbf{N}_{j}^{*}= & { }^{j} \mathbf{n}_{j, i}+{ }^{j} \mathbf{p}_{j c} \times{ }^{j} \mathbf{f}_{j, i}+\left({ }^{j} \mathbf{r}_{j, m}+{ }^{j} \mathbf{p}_{j c}\right) \\
& \times \mathrm{O}^{+}\left(t_{0, j, m} \pm k_{j, m} r_{j} \theta_{j, i} \pm k k_{j, m} r_{m} \theta_{m, i} \pm c_{j, m} r_{j} \dot{\theta}_{j, i} \pm c_{j, m} r_{m} \dot{\theta}_{m, i}\right)^{j} \mathbf{u}_{j, m}+\left({ }^{j} \mathbf{r}_{j, m+1}+{ }^{j} \mathbf{p}_{j c}\right) \\
& \times \mathrm{O}^{+}\left(t_{0, j, m+1} \pm k_{j, m+1} r_{j} \theta_{j, i+1} \pm k_{j, m+1} r_{m+1} \theta_{m+1, i+1} \pm c_{j, m+1} r_{j} \dot{\theta}_{j, i+1}\right. \\
& \left.\quad \pm c_{j, m+1} r_{m+1} \dot{\theta}_{m+1, i+1}\right)^{j} \mathbf{u}_{j, m+1}
\end{aligned}
$$

where ${ }^{j} \mathbf{u}_{j, m}$ and ${ }^{j} \mathbf{u}_{j, m+1}$ are the unit vectors of $\mathbf{t}_{j, m}$ and $\mathbf{t}_{j, m+1}$ acting on link $j$, and can be determined from Eqs. (20c) and (20d). ${ }^{j} \mathbf{F}_{j}^{*}$ and ${ }^{j} \mathbf{N}_{j}^{*}$ are the inertia force and moment of link $j$, and can be computed similarly by Eqs. (24a) and (24b).

The tendon force $t_{j, j+1}$ in Eqs. (25a) and (25b) can be computed form the moment balance equation of the upper level intermediate link. Once the joint forces and the moment of upper level links are solved, the unknown forces and moments in Eqs. (25a) and (25b) can be solved associated with the unknown forces and moments in the primary link. We also note that any vector defined in its local coordinate system on the intermediate link can be related to the coordinate system of its corresponding primary link by (19), (20) and (22).

We have derived the basic equations for the dynamic analysis of articulated, tendon-driven robotic mechanisms. The derivation of dynamic equations of the articulated, tendon-driven robotic devices will be described. Two examples will be used for illustration.

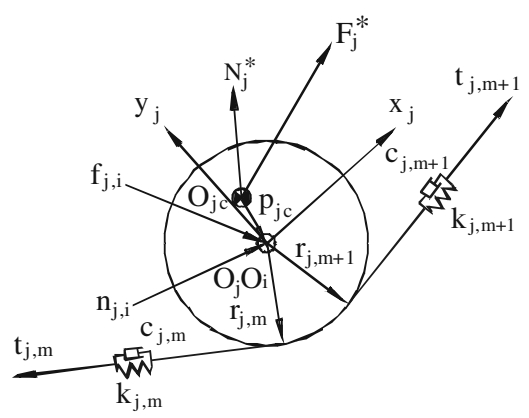

Fig. 6. A typical intermediate link $j$. 


\section{Examples}

\subsection{Example 1. A Robotic manipulator driven by flexible tendons}

Fig. 7 shows a two-jointed, four-link robotic manipulator driven by three open-ended tendons. The tendons are spooled to the motors located at the base link 0 . In this system, each segment of tendon between two rigid links is flexible with certain spring constant and damping coefficient. The vectors required for the kinematics are summarized in Table 1, while the vectors for the tendon forces are in Tables 2 and 3.

Thus, the force and moment balance equations for each link can be derived from Eqs. (23a), (23b), (25a) and (25b) as
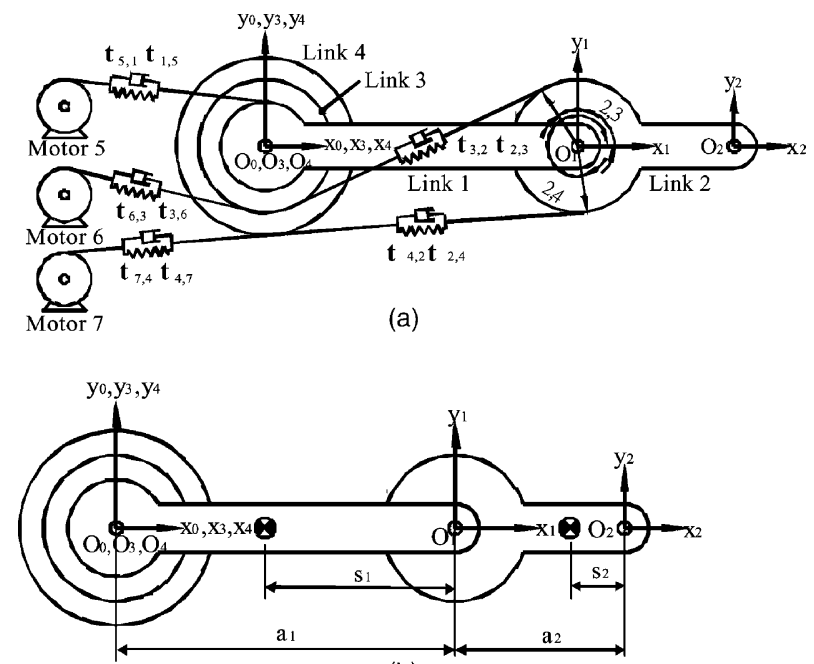

(b)

Fig. 7. (a) A robotic manipulator driven by flexible tendons and (b) kinematics of (a).

Table 1

Kinematics of the robotic mechanism in Example 1

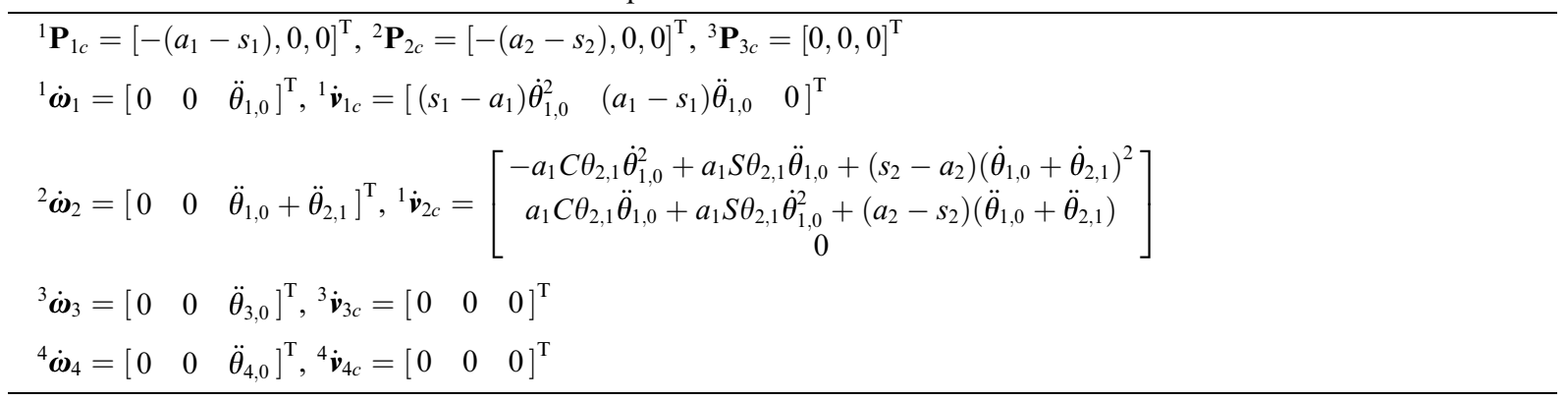


Table 2

The magnitudes of tendon forces for Example 1

\begin{aligned} \hline$t_{2,3} & =\mathrm{O}^{+}\left[t_{0,2,3}-k_{2,3}\left(r_{2} \theta_{2,1}+r_{3} \theta_{3,1}\right)-c_{2,3}\left(r_{2} \dot{\theta}_{2,1}+r_{3} \dot{\theta}_{3,1}\right)\right] \\ t_{2,4} & =\mathrm{O}^{+}\left[t_{0,2,4}+k_{2,4}\left(r_{2} \theta_{2,1}-r_{4} \theta_{4,1}\right)+c_{2,4}\left(r_{2} \dot{\theta}_{2,1}-r_{4} \dot{\theta}_{4,1}\right)\right] \\ t_{3,2} & =\mathrm{O}^{+}\left[t_{0,3,2}-k_{3,2}\left(r_{2} \theta_{2,1}+r_{3} \theta_{3,1}\right)-c_{3,2}\left(r_{2} \dot{\theta}_{2,1}+r_{3} \dot{\theta}_{3,1}\right)\right] \\ t_{3,6} & =\mathrm{O}^{+}\left[t_{0,3,6}+k_{3,6}\left(r_{3} \theta_{3,0}+r_{6} \theta_{6,0}\right)+c_{3,6}\left(r_{3} \dot{\theta}_{3,0}+r_{6} \dot{\theta}_{6,0}\right)\right] \\ t_{4,2} & =\mathrm{O}^{+}\left[t_{0,4,2}+k_{4,2}\left(r_{2} \theta_{2,1}-r_{4} \theta_{4,1}\right)+c_{4,2}\left(r_{2} \dot{\theta}_{2,1}-r_{4} \dot{\theta}_{4,1}\right)\right] \\ t_{4,7} & =\mathrm{O}^{+}\left[t_{0,4,7}+k_{4,7}\left(r_{4} \theta_{4,0}+r_{7} \theta_{7,0}\right)+c_{4,7}\left(r_{4} \dot{\theta}_{4,0}+r_{7} \dot{\theta}_{7,0}\right)\right] \\ t_{1,5} & =\mathrm{O}^{+}\left[t_{0,1,5}-k_{1,5}\left(r_{1} \theta_{1,0}-r_{5} \theta_{5,0}\right)-c_{1,5}\left(r_{1} \dot{\theta}_{1,0}-r_{5} \dot{\theta}_{5,0}\right)\right]\end{aligned}$

Table 3

The unit vectors of tendon forces for Example 1

$\begin{array}{rll}{ }^{2} \mathbf{u}_{2,3} & ={ }^{2} R_{1} \cdot\left[\begin{array}{lll}-S \beta_{2,3} & C \beta_{2,3} & 0\end{array}\right]^{\mathrm{T}} & { }^{2} \mathbf{u}_{2,4}={ }^{2} R_{1} \cdot\left[\begin{array}{lll}S \beta_{2,4} & -C \beta_{2,4} & 0\end{array}\right]^{\mathrm{T}} \\ { }^{3} \mathbf{u}_{3,2} & ={ }^{3} R_{1} \cdot\left[\begin{array}{lll}-S \beta_{3,2} & C \beta_{3,2} & 0\end{array}\right]^{\mathrm{T}} & { }^{3} \mathbf{u}_{3,6}={ }^{3} R_{0} \cdot\left[\begin{array}{llll}S \beta_{3,6} & -C \beta_{3,6} & 0\end{array}\right]^{\mathrm{T}} \\ { }^{4} \mathbf{u}_{4,2} & ={ }^{4} R_{1} \cdot\left[\begin{array}{lll}-S \beta_{4,2} & C \beta_{4,2} & 0\end{array}\right]^{\mathrm{T}} & { }^{4} \mathbf{u}_{4,7}={ }^{4} R_{0} \cdot\left[\begin{array}{lll}S \beta_{4,7} & -C \beta_{4,7} & 0\end{array}\right]^{\mathrm{T}} \\ { }^{1} \mathbf{u}_{1,5}={ }^{1} R_{0} \cdot\left[\begin{array}{lll}-S \beta_{1,5} & C \beta_{1,5} & 0\end{array}\right]^{\mathrm{T}} & \end{array}$

Link 2

$$
\begin{aligned}
& \mathbf{f}_{2,1}+\mathbf{t}_{2,3}+\mathbf{t}_{2,4}=\mathbf{F}_{2}^{*} \\
& \mathbf{p}_{2 c} \times \mathbf{f}_{2,1}+\left(\mathbf{r}_{2,3}+\mathbf{p}_{2 c}\right) \times \mathbf{t}_{2,3}+\left(\mathbf{r}_{2,4}+\mathbf{p}_{2 c}\right) \times \mathbf{t}_{2,4}=\mathbf{N}_{2}^{*}
\end{aligned}
$$

Link 3

$$
\begin{aligned}
& \mathbf{f}_{3,0}+\mathbf{t}_{3,2}+\mathbf{t}_{3,6}=\mathbf{F}_{3}^{*} \\
& \mathbf{p}_{3 c} \times \mathbf{f}_{3,0}+\left(\mathbf{r}_{3,2}+\mathbf{p}_{3 c}\right) \times \mathbf{t}_{3,2}+\left(\mathbf{r}_{3,6}+\mathbf{p}_{3 c}\right) \times \mathbf{t}_{3,6}=\mathbf{N}_{3}^{*}
\end{aligned}
$$

Link 4

$$
\begin{aligned}
& \mathbf{f}_{4,0}+\mathbf{t}_{4,2}+\mathbf{t}_{4,7}=\mathbf{F}_{4}^{*} \\
& \mathbf{p}_{4 c} \times \mathbf{f}_{4,0}+\left(\mathbf{r}_{4,2}+\mathbf{p}_{4 c}\right) \times \mathbf{t}_{4,2}+\left(\mathbf{r}_{4,7}+\mathbf{p}_{4 c}\right) \times \mathbf{t}_{4,7}=\mathbf{N}_{4}^{*}
\end{aligned}
$$

Link 1

$$
\begin{aligned}
& \mathbf{f}_{1,0}+\mathbf{t}_{1,5}=\mathbf{F}_{1}^{*}+\mathbf{f}_{2,1} \\
& \mathbf{p}_{1 c} \times \mathbf{f}_{1,0}+\left(\mathbf{r}_{1,5}+\mathbf{p}_{1 c}\right) \times \mathbf{t}_{1,5}=\mathbf{N}_{1}^{*}+\mathbf{r}_{1 c, 2} \times \mathbf{f}_{2,1}
\end{aligned}
$$

Expressing the force $\mathbf{f}_{2,1}$ in Eq. (26a) in terms of tendon forces and substituting into Eq. (26b), the moment balance equation of link 2 could be obtained as:

$$
\begin{aligned}
I_{2}\left(\ddot{\theta}_{1,0}+\ddot{\theta}_{2,1}\right)= & -m_{2}\left(a_{2}-s_{2}\right)\left[a_{1}\left(S \theta_{2,1} \dot{\theta}_{1,0}^{2}+C \theta_{2,1} \ddot{\theta}_{1,0}\right)+\left(a_{2}-s_{2}\right)\left(\ddot{\theta}_{1,0}+\ddot{\theta}_{2,1}\right)\right] \\
+ & r_{2}\left\{\mathrm{O}^{+}\left[t_{0,2,3}-k_{2,3}\left(r_{2} \theta_{2,1}-r_{3}\left(\theta_{1,0}-\theta_{3,0}\right)\right)-c_{2,3}\left(r_{2} \dot{\theta}_{2,1}-r_{3}\left(\dot{\theta}_{1,0}-\dot{\theta}_{3,0}\right)\right)\right]\right. \\
& \left.\quad-\mathrm{O}^{+}\left[t_{0,2,4}+r_{2}\left(k_{2,4} \theta_{2,1}+c_{2,3} \dot{\theta}_{2,1}\right)+r_{4}\left(k_{2,4}\left(\theta_{1,0}-\theta_{4,0}\right)+c_{2,3}\left(\dot{\theta}_{1,0}-\dot{\theta}_{4,0}\right)\right)\right]\right\}
\end{aligned}
$$


Similarly, the moment balance equations of links 3,4 , and 1 could be respectively obtained as:

$$
\begin{aligned}
I_{3} \ddot{\theta}_{3,0}= & r_{3}\left\{\mathrm{O}^{+}\left[t_{0,3,2}-k_{3,2}\left(r_{2} \theta_{2,1}-r_{3}\left(\theta_{1,0}-\theta_{3,0}\right)\right)-c_{3,2}\left(r_{2} \dot{\theta}_{2,1}-r_{3}\left(\dot{\theta}_{1,0}-\dot{\theta}_{3,0}\right)\right)\right]\right. \\
& \left.-\mathrm{O}^{+}\left[t_{0,3,6}+k_{3,6}\left(r_{3} \theta_{3,0}+r_{6} \theta_{6,0}\right)+c_{3,6}\left(r_{3} \dot{\theta}_{3,0}+r_{6} \dot{\theta}_{6,0}\right)\right]\right\} \\
I_{4} \ddot{\theta}_{4,0}= & r_{4}\left\{\mathrm{O}^{+}\left[t_{0,4,2}+k_{4,2}\left(r_{2} \theta_{2,1}+r_{4}\left(\theta_{1,0}-\theta_{4,0}\right)\right)+c_{4,2}\left(r_{2} \dot{\theta}_{2,1}+r_{4}\left(\dot{\theta}_{1,0}-\dot{\theta}_{4,0}\right)\right)\right]\right. \\
& \left.\quad-\mathrm{O}^{+}\left[t_{0,4,7}+k_{4,7}\left(r_{4} \theta_{4,0}+r_{7} \theta_{7,0}\right)+c_{4,7}\left(r_{4} \dot{\theta}_{4,0}+r_{7} \dot{\theta}_{7,0}\right)\right]\right\} \\
I_{1} \ddot{\theta}_{1,0}= & -\left(r_{2}+r_{3}\right) \mathrm{O}^{+}\left[t_{0,2,3}-k_{2,3}\left(r_{2} \theta_{2,1}-r_{3}\left(\theta_{1,0}-\theta_{3,0}\right)\right)-c_{2,3}\left(r_{2} \dot{\theta}_{2,1}-r_{3}\left(\dot{\theta}_{1,0}-\dot{\theta}_{3,0}\right)\right)\right] \\
& +\left(r_{2}-r_{4}\right) \mathrm{O}^{+}\left[t_{0,2,4}+k_{2,4}\left(r_{2} \theta_{2,1}+r_{4}\left(\theta_{1,0}-\theta_{4,0}\right)\right)+c_{2,4}\left(r_{2} \dot{\theta}_{2,1}+r_{4}\left(\dot{\theta}_{1,0}-\dot{\theta}_{4,0}\right)\right)\right] \\
+ & r_{1} \mathrm{O}^{+}\left[t_{0,1,5}+k_{1,5}\left(-r_{1} \theta_{1,0}+r_{5} \theta_{5,0}\right)+c_{1,5}\left(-r_{1} \dot{\theta}_{1,0}+r_{5} \dot{\theta}_{5,0}\right)\right]-a_{1}^{2}\left(m_{1}+m_{2}\right) \ddot{\theta}_{1,0} \\
- & m_{1} S_{1}^{2} \ddot{\theta}_{1,0}+a_{1}\left\{2 m_{1} s_{1} \ddot{\theta}_{1,0}+m_{2}\left(a_{2}-s_{2}\right)\left[S \theta_{2,1}\left(\dot{\theta}_{1,0}+\dot{\theta}_{2,1}\right)^{2}-C \theta_{2,1}\left(\ddot{\theta}_{1,0}\right.\right.\right. \\
& \left.\left.\left.+\ddot{\theta}_{2,1}\right)\right]\right\}
\end{aligned}
$$

Eqs. (27)-(30) are the equations of motion of the system. Once the equations of motion are solved, the tension in each segment of tendon can be calculated via the equations in Table 2.

\subsection{Example 2. Control of the one-DOF manipulator driven by two actuators}

In this example, a one-DOF device is used to demonstrate the difference in control performance with and without the $\mathrm{O}^{+}$operator acting on the tendons. As shown in Fig. 8, the one-DOF manipulator is driven by two flexible tendons spooled to the actuators on the ground. A simple "PD feedback" control technique is implemented for controlling the manipulator. Following the same procedure illustrated in Example 1, the dynamic equation can be concluded as:

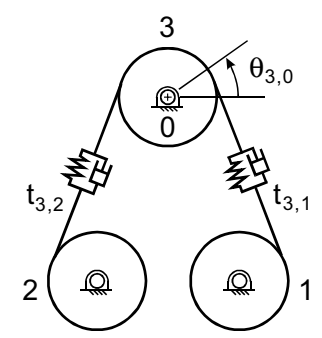

Fig. 8. One-DOF manipulator driven by two actuators.

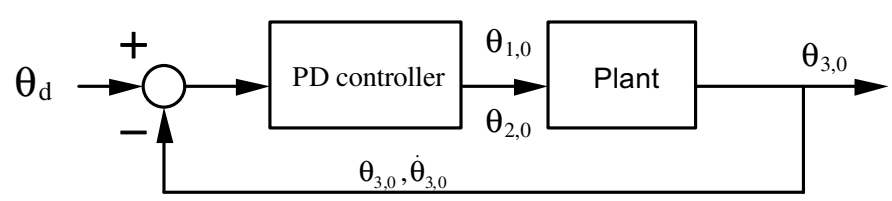

Fig. 9. Block diagram of the PD control system. 


$$
\begin{aligned}
I_{3} \ddot{\theta}_{3,0}= & -r_{3} \mathrm{O}^{+}\left[t_{0,3,1}+K_{3,1}\left(r_{3} \theta_{3,0}-r_{1} \theta_{1,0}\right)+C_{3,1}\left(r_{3} \dot{\theta}_{3,0}-r_{1} \dot{\theta}_{1,0}\right)\right] \\
& +r_{3} \mathrm{O}^{+}\left[t_{0,3,2}-K_{3,2}\left(r_{3} \theta_{3,0}-r_{2} \theta_{2,0}\right)-C_{3,2}\left(r_{3} \dot{\theta}_{3,0}-r_{2} \dot{\theta}_{2,0}\right)\right]
\end{aligned}
$$

and the inputs are designed as

$$
\begin{aligned}
& \theta_{1,0}=K_{P}\left(\theta_{d}-\theta_{3,0}\right)+K_{D}\left(\dot{\theta}_{d}-\dot{\theta}_{3,0}\right) \\
& \theta_{2,0}=K_{P}\left(\theta_{d}-\theta_{3,0}\right)+K_{D}\left(\dot{\theta}_{d}-\dot{\theta}_{3,0}\right)
\end{aligned}
$$

Fig. 9 shows the block diagram of the control system. The designed output of $\theta_{3,0}$ is a sinusoidal function $\sin (t)$. The dynamic system is simulated and the results are shown as follows.

Fig. 10 shows the motion responses of the output with and without the rectifier $\mathrm{O}^{+}$in the dynamic equation. The results of the outputs will depend on the stiffness of tendon as well as the proportional and derivative gains tuned for the controller. The higher the stiffness of tendons is, the more similar outputs of the two models become. The tendon force in each tendon is also plotted in Fig. 11a and $\mathrm{b}$ where the pretension $t_{0}$ is set to $0.5 \mathrm{~N}$. It can be seen that negative values appears in the tendon force curves for the system without the rectifier $\mathrm{O}^{+}$in the equation. This result does not comply with the force transmission characteristics of tendons. Thus, if the tendon force is required for the force control simulation, there may cause errors for the results.

To compare the performance of the system under different pretension, the system is simulated with $t_{0}=0.5 \mathrm{~N}$ and $3.5 \mathrm{~N}$. Fig. 12 shows the motion response of the output while Figs. 13 and 14 show the responses of tension in tendons. Comparing the tendon force curves shown in Figs. 13 and 14, it can be noted that the higher the pretension is, the stiffer the system becomes. Also, note that the tendons with $t_{0}=0.5 \mathrm{~N}$ reached zero tension state during the operation. Thus, it can be concluded that if pretensioning is not well managed, slackness may occur in tendons during system modeling and results in errors. Therefore, the pretension of tendons may play an important role in the dynamic response of tendon-driven manipulators.

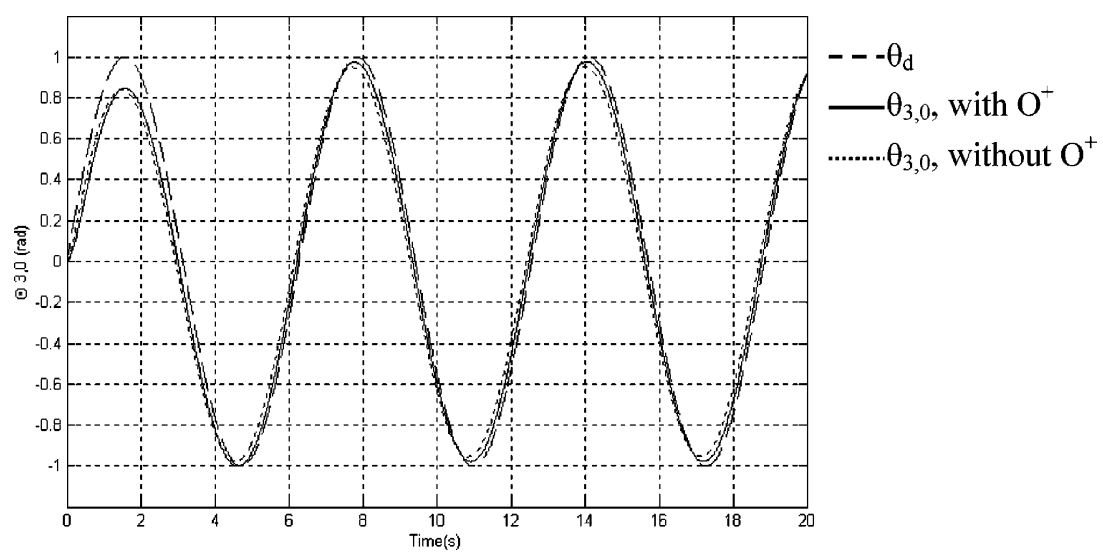

Fig. 10. Comparison of $\theta_{3,0}$ with and without $\mathrm{O}^{+}$. 

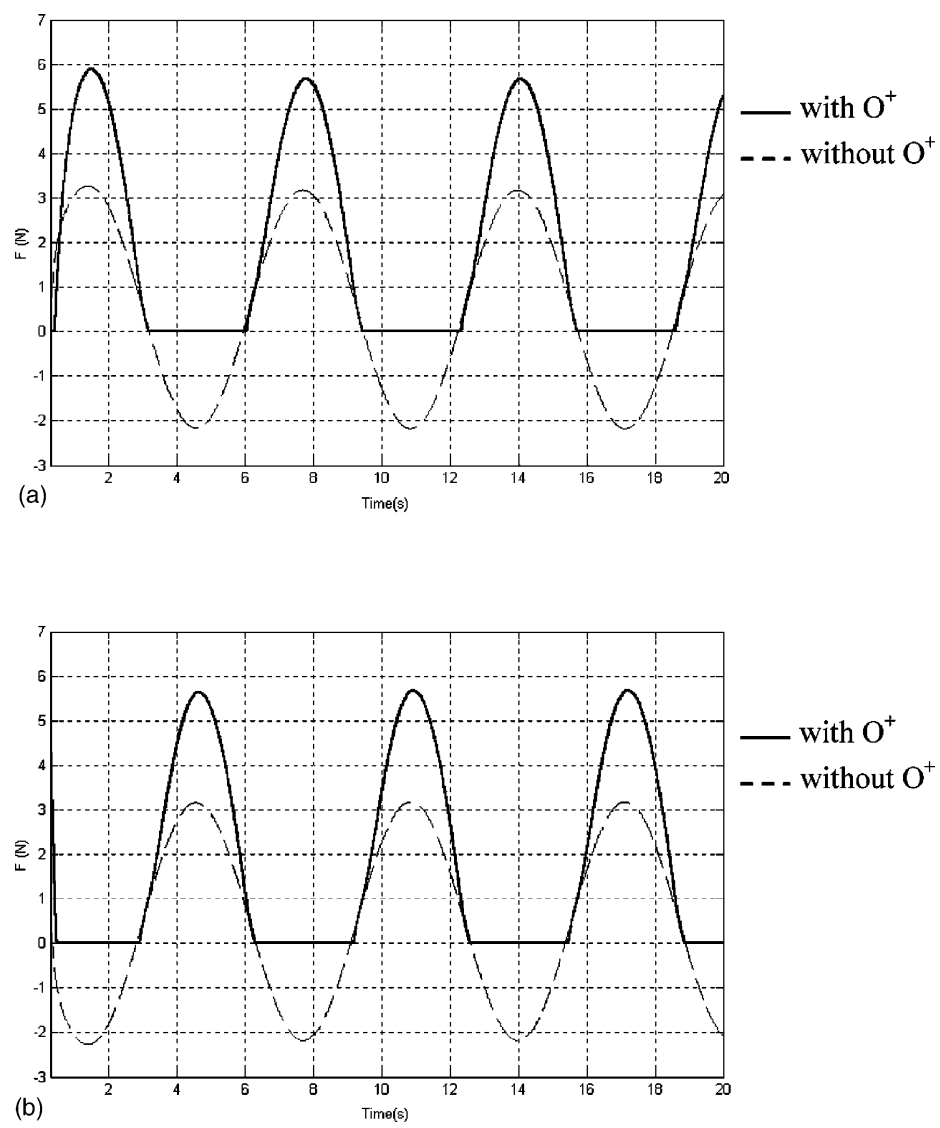

Fig. 11. Comparison of tendon force (a) $t_{3,1}$ and (b) $t_{3,2}$ with and without $\mathrm{O}^{+}$.

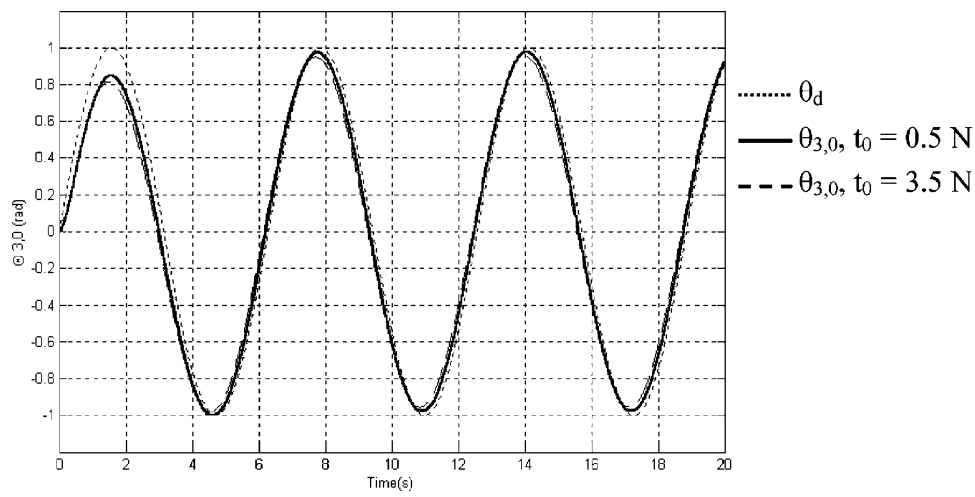

Fig. 12. Responses of $\theta_{3,0}$ under different pretensions. 


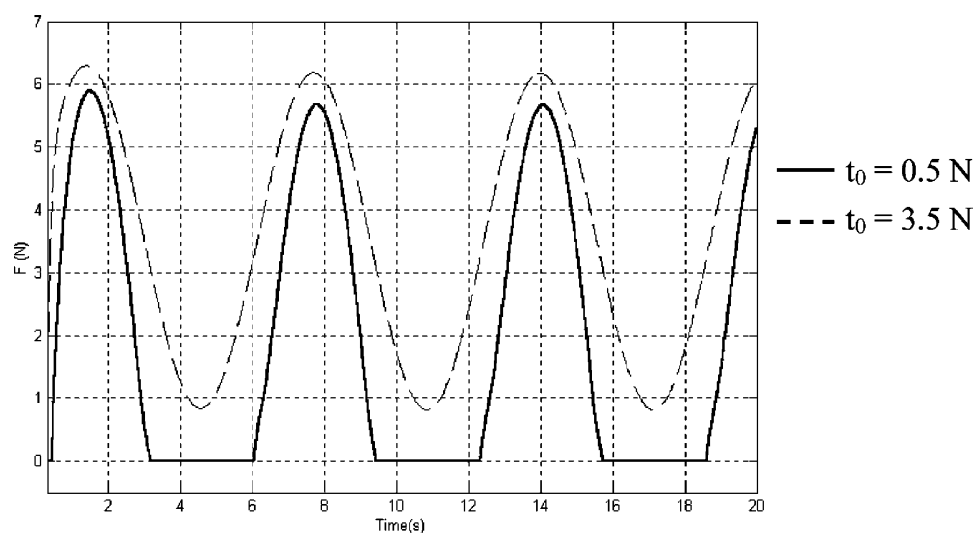

Fig. 13. Responses of $t_{3,1}$ with different pretensions.

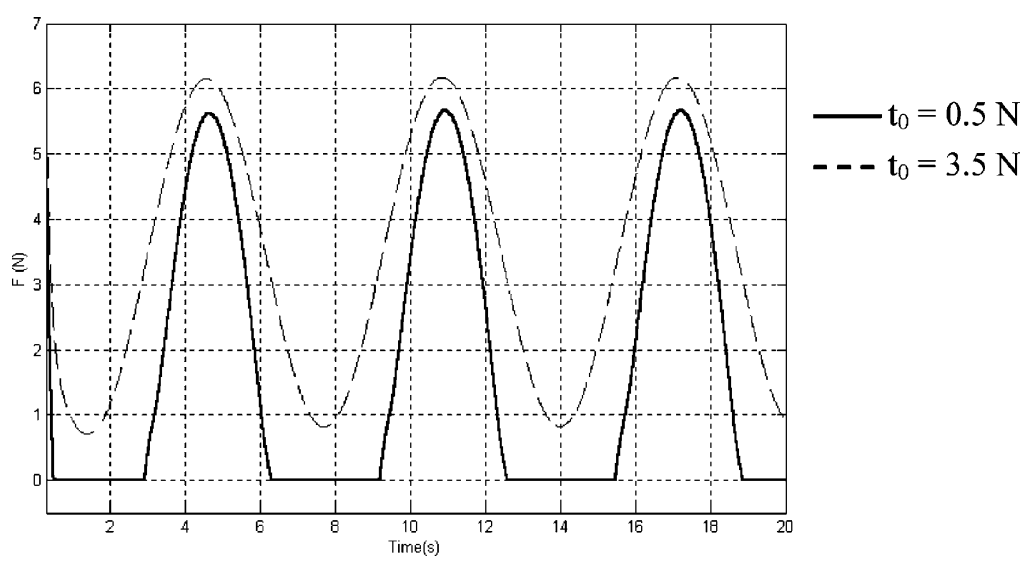

Fig. 14. Responses of $t_{3,2}$ with different pretensions.

\section{Conclusion}

A systematic methodology for the dynamic analysis of tendon-driven robotic mechanisms with flexible tendons is developed. A novel representation of the tendon force is introduced. The $\mathrm{O}^{+}$operator is used to represent the force transmission characteristics of tendons. The method subsequently uses the recursive algorithm to calculate the dynamics of links. Tendon forces, reaction forces and compliance effects are all derived by the methods developed in this research.

The performance of such system is also studied by implementing a PD controller onto the oneDOF system. The results of the system output with and without the operator in the equation are compared. It is also shown that the magnitude of pretension of tendons may play an important role in the dynamic response of tendon-driven manipulators. 


\section{References}

[1] J.K. Salisbury, Kinematic and Force Analysis of Articulated Hands, Ph.D. Dissertation, Department of Mechanical Engineering, Stanford University, Stanford, CA, 1982.

[2] S.C. Jacobsen, J.E. Wood, D.F. Knutti, K.B. Biggers, The Utah-MIT dexterous hand: work in progress, Int. J. Rob. Res. 3 (4) (1985) 21-50.

[3] J. Albus, R. Bostelman, N. Dagalakis, The NIST spider, A robot crane, J. Res. NIST 97 (3) (1992) 373-385.

[4] S. Kawamura, W. Choe, S. Tanaka, S.R. Pandian, Development of an ultrahigh speed robot FALCON using wire drive system, in: IEEE International Conference On Robotics and Automation, 1995, pp. 215-220.

[5] A. Morecki, Z. Busko, H. Gasztold and K. Jaworek, Synthesis and control of the anthropomorphic two-handed manipulator, in: Proceedings of the 10th International Symposium on Industrial Robots, 1980, pp. 461-474.

[6] L.W. Tsai, J.J. Lee, Kinematic analysis of tendon-driven robotic mechanisms using graph theory, ASME J. Mech. Transmissions Automat. Des. 111 (1) (1989) 59-65.

[7] M.G. Hollars, R.H. Cannon, Initial experiments on the end-point control of a two-link manipulator with flexible tendons, in: ASME Winter Annual Meeting, Miami, FL, 1985.

[8] S.L. Chang, Compliance and dynamic analysis of tendon-driven robotic mechanisms, in: ASME, Proceedings of the 19th Annual ASME Design Automation Conference, Part 1, 1993, pp. 229-233.

[9] G.M. Prisco, M. Bergamasco, Dynamic modeling of a class of tendon driven manipulators, in: Proceedings of the IEEE International Conference Robotics Automation, Monterey, 1997.

[10] S.C. Jacobsen, H. Ko, E.K. Iversen, C.C. Davis, Antagonistic control of a tendon-driven manipulator, in: Proceedings of the IEEE International Conference on Robotics Automation, 1989.

[11] C.R. Johnstun, C.C. Smith, Modeling and design of a mechanical tendon actuation systems, ASME J. Dyn. Syst. Meas. Control 114 (1992) 253.

[12] L.W. Tsai, Robot Analysis-The Mechanics of Serial and Parallel Manipulators, John Wiley \& Sons, 1999.

[13] J. Denavit, R.S. Hartenberg, A kinematic notation for lower pair mechanisms based on matrices, ASME J. Appl. Mech. 77 (1955) 215-221.

[14] D.E. Orin, R.B. McGhee, M. Vukobratovic, G. Hartoch, Kinematic and kinetic analysis of open-chain linkage utilizing Newton-Euler methods, Math. Biosci. 43 (1979) 107-130.

[15] M. Kaneko, W. Pactsch, H. Tolle, Input-dependent stability of joint torque control of tendon driven robot hands, IEEE Trans. Ind. Electron. 39 (2) (1992).

[16] J.J. Lee, L.W. Tsai, Torque resolver design for tendon-driven manipulators, ASME J. Mech. Des. 115 (4) (1993) 877-883. 